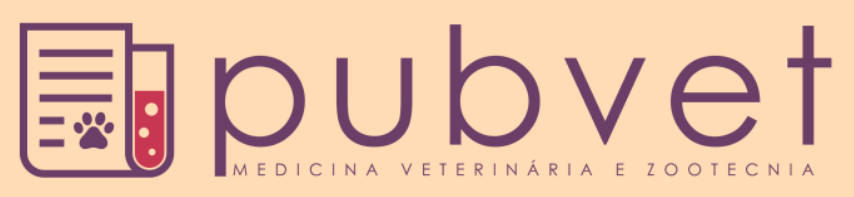

HTTP://DX.DOI.ORG/10.22256/PUBVET.V11N11.1169-1174

\title{
Produtividade de forragem, composição química e morfogênese de Megathyrsus maximus cv. Mombaça sob períodos de descanso
}

\author{
Newton de Lucena Costa ${ }^{*}$, Liana Jank ${ }^{2}$, João Avelar Magalhães ${ }^{3}$, Antônio Neri Azevedo \\ Rodrigues $^{4}$, Fabíola Helena dos Santos Fogaça ${ }^{5}$, Amaury Burlamaqui Bendahan', \\ Francisco José de Seixas Santos 6
}

\author{
${ }^{1}$ Eng. Agr., D.Sc., Pesquisador da Embrapa Roraima, Boa Vista, RR. \\ ${ }^{2}$ Eng. Agr., Ph.D., Pesquisadora da Embrapa Gado de Corte, Campo Grande, MS. \\ ${ }^{3}$ Méd. Vet., Pesquisador da Embrapa Meio-Norte, Parnaíba, PI. \\ ${ }^{4}$ Eng. Agr., D.Sc., Professor do Instituto Federal de Rondônia, Colorado do Oeste, RO. \\ ${ }^{5}$ Zootecnista, D.Sc., Pesquisadora da Embrapa Meio-Norte, Parnaíba, PI. \\ ${ }^{6}$ Eng. Agr., D.Sc., Pesquisador da Embrapa Meio-Norte, Parnaíba, PI. \\ *Autor para correspondência, E-mail: newtonlucena@yahoo.com.br
}

\begin{abstract}
RESUMO. O efeito do período de descanso (14, 21, 28, 35, 42 e 49 dias) sobre a produção e composição química da forragem e características morfogênicas e estruturais de Megathyrsus maximus cv. Mombaça foi avaliado em condições de campo. $\mathrm{O}$ aumento do período de descanso resultou em maiores rendimentos de matéria seca verde (MSV) e vigor de rebrota, contudo, implicou em decréscimos significativos dos teores de nitrogênio, fósforo, cálcio, magnésio e potássio. As taxas de aparecimento e expansão de folhas são inversamente proporcionais aos períodos de descanso, ocorrendo o inverso quanto ao comprimento médio de folhas (CMF), índice de área foliar (IAF) e taxa de senescência foliar. Os maiores rendimentos de MSV, vigor de rebrota, número de folhas vivas perfilho${ }^{1}$, CMF e IAF foram obtidos aos 36,4; 39,2; 40,8; 39,6 e 41,3 dias de rebrota. O período de descanso mais adequado para pastagens de M. maximus $\mathrm{cv}$. Mombaça, visando a conciliar produção, vigor de rebrota e qualidade da forragem, situa-se entre 35 e 42 dias.
\end{abstract}

Palavras-chave: composição química, folhas, matéria seca, perfilhamento, senescência

\section{Forage yield, chemical composition and morphogenesis of Megathyrsus maximus cv. Mombaça under rest periods}

\begin{abstract}
The effects of rest periods (14, 21, 28, 35, 42 and 49 days) on green dry matter (GDM) yield, chemical composition and morphogenetic and structural characteristics of Megathyrsus maximus cv. Mombaça were evaluated under natural field conditions. GDM yields and regrowth increased consistently with highest rest periods. The leaf appearance and leaf expansion rates are inversely proportional to the rest periods, occurring the inverse for average leaf length, leaf area index and foliar senescence rate. Maximum GDM yields, regrowth yields, number of leaves tiller ${ }^{-1}$, average leaf length and leaf area index were obtained with rest periods at $36.4 ; 39.2 ; 40.8 ; 39.6$ and 41.3 days. These data suggest that grass grazing at 35 to 42 days of rest period were optimal for obtain maximum yields and regrowth of rich forage.
\end{abstract}

Keywords: chemical composition, dry matter, leaves, tillering, senescence 


\title{
Rendimiento de forraje, composición química y morfogénesis de Megathyrsus maximus cv. Mombaça bajo periodos de descanso
}

\begin{abstract}
RESUMEN. El efecto del período de descanso (14, 21, 28, 35, 42 y 49 días) en la producción, composición química del forraje y las características morfogenéticas y estructurales de Megathyrsus maximus cv. Mombasa se evaluó en condiciones de campo. Aumentar el período de descanso resultó en mayores rendimientos de materia seca verde (MSV) y el vigor de rebrote, sin embargo, resultó en reducciones significativas de los contenidos de nitrógeno, fósforo, calcio, magnesio y potasio. Las tasas de aparición y la expansión de las hojas son inversamente proporcional a los períodos de descanso, mientras que lo contrario ocurrió para la longitud media de las hojas (LMF), el índice de área foliar (IAF) y de la tasa de senescencia de las hojas. Los mayores rendimientos de MSV, vigor de rebrote, número de hojas por macollas, LMF y IAF se obtuvieron a 36,4; 39,2; 40,8; 39,6 y 41,3 días de rebrote. El período de descanso más adecuado para el pastoreo de pasturas de M. maximus cv. Mombasa, destinada a conciliar la producción, el vigor de rebrote y la calidad del forraje, está entre 35 y 42 días de descanso.
\end{abstract}

Palabras clave: composición química, hojas, macollaje, materia seca, senescencia

\section{Introdução}

Em Roraima, a pecuária é uma atividade econômica em franca expansão e as pastagens cultivadas representam o mais importante recurso forrageiro para a alimentação dos rebanhos. A utilização do pastejo contínuo ou períodos mínimos de descanso e altas intensidades de desfolhação são fatores que contribuem para baixa disponibilidade e qualidade da forragem, com reflexos negativos nos índices de desempenho zootécnico dos animais. A produtividade da pastagem é fortemente influenciada pelas condições ambientais (temperatura, luz, água e fertilidade do solo) e práticas de manejo, enquanto que sua perenidade decorre, entre outros fatores, da capacidade de reconstituição e manutenção da área foliar após a desfolhação, a qual afeta a estrutura do dossel, determinando sua velocidade de crescimento, acúmulo de forragem, composição química e persistência (Nabinger and Pontes, 2002). O período de descanso disponibilizado para o crescimento da pastagem afeta sua produtividade, composição química, capacidade de rebrota e persistência. Pastejos frequentes proporcionam maiores rendimentos de forragem, porém, concomitantemente, ocorrem decréscimos acentuados em sua composição química, com maior acúmulo de material fibroso, decréscimo na relação folha/colmo e, consequentemente, menor consumo pelos animais (Costa, 2004, Lemaire et al., 2011).

No manejo adequado de pastagens busca-se o equilíbrio entre produção e qualidade da forragem, visando assegurar os requerimentos nutricionais dos animais e garantindo, simultaneamente, a persistência e a produtividade das pastagens. A produtividade das gramíneas forrageiras decorre da contínua emissão de folhas e perfilhos, processo que assegura a restauração da área foliar após o pastejo e a perenidade da pastagem. Os processos de formação e desenvolvimento de folhas são fundamentais para o crescimento vegetal, considerando-se a importância das folhas no processo de fotossíntese, ponto de partida para a formação de novos tecidos (Santos et al., 2012). A morfogênese de gramíneas forrageiras durante seu crescimento vegetativo é caracterizada por três fatores: a taxa de aparecimento, a taxa de alongamento e a longevidade das folhas. A taxa de aparecimento e a longevidade das folhas determinam o número de folhas vivas/perfilho, as quais são geneticamente determinadas e podem ser afetadas pelos fatores ambientais e as práticas de manejo adotadas (Nabinger and Pontes, 2002, Lemaire et al., 2011).

Neste trabalho foram avaliados os efeitos do período de descanso sobre a produção de forragem, vigor de rebrota, composição química e características morfogênicas e estruturais de Megathyrsus maximus cv. Mombaça nos cerrados de Roraima.

\section{Material e Métodos}

O ensaio foi conduzido no Campo Experimental da Embrapa Roraima, localizado em Boa Vista, durante o período de maio a setembro de 2014, o qual correspondeu a uma precipitação acumulada de $865,4 \mathrm{~mm}$ e temperatura média mensal de $24,9^{\circ} \mathrm{C}$. O solo da área experimental é 
Latossolo Amarelo, textura média, com as seguintes características químicas, na profundidade de $0-20 \mathrm{~cm}: \mathrm{pH}_{\mathrm{H} 2 \mathrm{O}}=5,3 ; \mathrm{P}=4,1$ $\mathrm{mg} / \mathrm{kg} ; \mathrm{Ca}+\mathrm{Mg}=0,98 \mathrm{cmol}_{\mathrm{c}} \cdot \mathrm{dm}^{-3} ; \mathrm{K}=0,08$ $\mathrm{cmol}_{c} \cdot \mathrm{dm}^{-3} ; \mathrm{Al}=0,35 \mathrm{cmol}_{\mathrm{c}} \mathrm{dm}^{-3} ; \mathrm{H}+\mathrm{Al}=2,87$ $\mathrm{cmol}_{\mathrm{c}} \cdot \mathrm{dm}^{-3}$.

O delineamento experimental foi inteiramente casualizado com três repetições. Os tratamentos consistiram de seis períodos de descanso $(14,21$, $28, \quad 35,42$ e 49 dias). A adubação de estabelecimento constou da aplicação de $90 \mathrm{~kg}$ de $\mathrm{N} \mathrm{ha}^{-1}, 50 \mathrm{~kg}$ de $\mathrm{P}_{2} \mathrm{O}_{5}$ ha $^{-1}$ e $60 \mathrm{~kg}_{\text {de }} \mathrm{K}_{2} \mathrm{O}$ ha ${ }^{-1}$, sob a forma de ureia, superfosfato triplo e cloreto de potássio, respectivamente. A adubação nitrogenada foi parcelada em duas vezes, sendo $2 / 3$ quando do plantio e $1 / 3$ decorridos 35 dias. $\mathrm{O}$ tamanho das parcelas foi de 2,0 x 2,0 m, sendo a área útil de $1,0 \mathrm{~m}^{2}$.

Os parâmetros avaliados foram rendimento de matéria seca verde (MSV), teores de nitrogênio $(\mathrm{N})$, fósforo $(\mathrm{P})$, cálcio $(\mathrm{Ca})$, magnésio $(\mathrm{Mg})$ e potássio $(\mathrm{K})$, número de folhas vivas perfilho ${ }^{-1}$ (NFV), taxa de aparecimento de folhas (TAF), taxa de expansão foliar (TEF), taxa de senescência foliar (TSF), comprimento médio de folhas (CMF) e índice de área foliar (IAF). A TEF e a TAF foram calculadas dividindo-se o comprimento acumulado de folhas e o número total de folhas no perfilho, respectivamente, pelo período de rebrota. O CMF foi determinado pela divisão do alongamento foliar total do perfilho pelo número de folhas. Para o cálculo da área foliar foram coletadas amostras de folhas verdes completamente expandidas, procurando-se obter uma área entre 200 e $300 \mathrm{~cm}^{2}$. As amostras foram digitalizadas e a área foliar estimada com o auxílio de planímetro ótico eletrônico (Li-Cor 3100C). Posteriormente, as amostras foram levadas à estufa com ar forçado a $65^{\circ} \mathrm{C}$ até atingirem peso constante, obtendo-se a MS foliar. A área foliar específica (AFE) foi determinada através da relação entre a área de folhas verdes e a sua MS $\left(\mathrm{m}^{2} / \mathrm{g}\right.$ MS foliar). O IAF foi determinado a partir do produto entre a MS total das folhas verdes ( $\mathrm{g}$ de $\left.\mathrm{MS} / \mathrm{m}^{2}\right)$ pela AFE $\left(\mathrm{m}^{2} / \mathrm{g}\right.$ de MS foliar). A TSF foi obtida dividindo-se o comprimento da folha que se apresentava de coloração amarelada ou necrosada pela idade de rebrota. $\mathrm{O}$ vigor de rebrota foi avaliado através da produção de MS aos 21 dias após o corte à idade do primeiro corte.

Os dados foram submetidos à análise de variância e de regressão considerando o nível de significância de 5\% de probabilidade. Para se estimar a resposta dos parâmetros avaliados aos períodos de descanso, a escolha dos modelos de regressão baseou-se na significância dos coeficientes linear e quadrático, por meio do teste "t", de Student, ao nível de 5\% de probabilidade.

\section{Resultados e Discussão}

Os rendimentos de MSV foram afetados pelos períodos de descanso $(\mathrm{P}<0,05)$, sendo a relação quadrática e o máximo valor estimado aos 36,4 dias de descanso (Tabela 1). Nos cerrados de Rondônia, para pastagens de M. maximus cvs. Massai, Tanzânia-1 e Vencedor, Costa et al. (2007) estimaram maiores rendimentos de forragem para períodos de descanso variando entre 28 e 35 dias. $\mathrm{O}$ efeito do período de descanso sobre o vigor de rebrota ajustou-se ao modelo quadrático de regressão e o máximo rendimento de MSV foi obtido aos 39,2 dias. Costa (2004) constatou máximo vigor de rebrota para pastagens de $M$. maximus cv. Centenário no período entre 21 e 35 dias após o corte das plantas. A velocidade de rebrota apresenta alta correlação com a preservação dos meristemas apicais, pois sua preservação favorece a formação de tecidos fotossintetizantes por meio da expansão de novas folhas, enquanto que com a remoção de meristemas apicais o novo crescimento é mais lento e tem origem a partir do desenvolvimento de gemas, notadamente de origem basal, para a produção de folhas (Difante et al., 2011, Cunha et al., 2012). Os teores de N, P, Ca Mg e K foram negativa e linearmente afetados pelos períodos de descanso (Tabela 1), evidenciando efeito de diluição de seus teores com o avanço do estádio de crescimento da gramínea. Os teores registrados neste trabalho foram semelhantes ou superiores aos relatados por Costa et al. (2007) para diversos genótipos de M. maximus cvs. Massai, Vencedor e Centenário, submetidos a diferentes períodos de descanso. Para o P, Ca, Mg e K, os teores obtidos com períodos de descanso de até 35 dias, foram superiores ao nível crítico interno determinados por Costa (2004) para M. maximus cv. Centenário $(1,89 ; 3,89 ; 2,87$ e $19,89 \mathrm{~g} / \mathrm{kg}$, respectivamente para P, Ca, Mg e K). Oliveira et al. (2009) constataram máximas concentrações de $\mathrm{N}, \mathrm{P}, \mathrm{K}$, $\mathrm{Ca}$ e $\mathrm{Mg}$, em M. maximus cv. Mombaça, respectivamente aos 104, 102, 105, 68 e 78 dias de descanso.

A TAF e a TEF foram negativa e linearmente afetadas pelo período de descanso, enquanto que para o NFV, CMF e IAF os ajustes foram 
quadráticos e os máximos valores obtidos aos 40,8; 39,6 e 41,3 dias de descanso, respectivamente (Tabela 2). Nos cerrados de Rondônia, em pastagens de M. maximus cvs. Massai, Vencedor e Centenário, Costa (2004) reportou maiores NFV, CMF e IAF para períodos compreendidos entre 28 e 35 dias de rebrota, os quais foram recomendados como mais adequados para o manejo das gramíneas. Em pastagens de M. maximus cv. Mombaça, o prolongamento do período de descanso comprometeu a estrutura de seu dossel, estreitando a relação folha/colmo e reduzindo a população de perfilhos, contudo, adaptações morfológicas e estruturais do dossel forrageiro asseguraram satisfatória rebrota sob manejo em que o período de descanso permite o aparecimento de 2,5 novas folhas perfilho ${ }^{-1}$, durante a época chuvosa. O período adequado de descanso em pastagens de capim-mombaça não deve exceder o tempo para aparecimento de três folhas adultas por perfilho (Cândido et al., 2005a, Cândido et al., 2005b, Gomide et al., 2007). O IAF representa a síntese das características morfogênicas e estruturais da gramínea e reflete o balanço dos processos que determinam a oferta (fotossíntese) e a demanda (respiração, acúmulo de reservas, síntese e senescência de tecidos) de fotoassimilados, estabelecendo o ritmo de crescimento da pastagem (Nabinger and Carvalho, 2009). Com o aumento do período de descanso, a percentagem de luz interceptada pelo dossel atinge seu ponto máximo, $\mathrm{IAF}_{\text {teto }}$, onde para cada nova folha surgida na porção superior da planta ocorre a senescência de uma folha em sua porção inferior, o que estabiliza ou reduz a disponibilidade de biomassa verde como decorrência da redução do coeficiente de extinção luminosa (Nabinger and Pontes, 2002).

Tabela 1. Produção de matéria seca verde (MSV - $\mathrm{kg} \mathrm{ha}^{-1}$ ), vigor de rebrota (VR - kg MSV/21 dias) e teores de nitrogênio $(\mathrm{N})$, fósforo $(\mathrm{P})$, cálcio $(\mathrm{Ca})$, magnésio $(\mathrm{Mg})$ e potássio $(\mathrm{K})$ de Megathyrsus maximus cv. Mombaça, em função do período de descanso. Médias de três cortes.

\begin{tabular}{|c|c|c|c|c|c|c|c|}
\hline \multirow{2}{*}{ Variáveis } & \multicolumn{6}{|c|}{ Períodos de Descanso (dias) } & \multirow{2}{*}{ Equação de Regressão } \\
\hline & 14 & 21 & 28 & 35 & 42 & 49 & \\
\hline MSV & 1.315 & 1.764 & 2.318 & 2.657 & 2.819 & 2.472 & $Y=1.105+216,37 X-2,9643 X^{2}\left(R^{2}=0,96\right)$ \\
\hline VR & 987 & 1.289 & 1.634 & 1.971 & 1.814 & 1.719 & $\mathrm{Y}=383+114,42 \mathrm{X}-1,4552 \mathrm{X}^{2}\left(\mathrm{R}^{2}=0,94\right)$ \\
\hline $\mathrm{N}(\mathrm{g} / \mathrm{kg})$ & 28,35 & 27,11 & 26,23 & 23,98 & 23,07 & 22,15 & $Y=30,98-0,1852 \times\left(r^{2}=0,89\right)$ \\
\hline $\mathrm{P}(\mathrm{g} / \mathrm{kg})$ & 2,34 & 2,08 & 1,97 & 1,81 & 1,74 & 1,71 & $Y=2,498-0,0177 \times\left(r^{2}=0,92\right)$ \\
\hline $\mathrm{Ca}(\mathrm{g} / \mathrm{kg})$ & 4,78 & 4,33 & 4,02 & 3,87 & 3,55 & 3,12 & $Y=5,332-0,0209 \times\left(r^{2}=0,93\right)$ \\
\hline $\mathrm{Mg}(\mathrm{g} / \mathrm{kg})$ & 3,67 & 3,32 & 3,14 & 2,78 & 2,59 & 2,21 & $Y=4,218-0,0402 \times\left(r^{2}=0,97\right)$ \\
\hline $\mathrm{K}(\mathrm{g} / \mathrm{kg})$ & 22,67 & 20,39 & 19,11 & 18,47 & 18,09 & 16,89 & $Y=23,95-0,1487 \times\left(r^{2}=0,97\right)$ \\
\hline
\end{tabular}

O CMF é a característica plástica mais responsiva à intensidade e frequência de desfolhação e considerada como a principal estratégia morfológica de escape das plantas ao pastejo (Pedreira et al., 2009, Lemaire et al., 2011) As gramíneas sob desfolhações frequentes podem apresentar abundante perfilhamento, hábito de crescimento prostrado e elevado ritmo de expansão foliar, possibilitando maior interceptação de luz e rebrotas mais vigorosas (Nabinger and Pontes, 2002). As TAF, TEF e CMF obtidas neste trabalho, independentemente dos períodos de descanso, foram inferiores às reportadas por Macedo et al. (2010) avaliando $M$. maximus cv. Mombaça que constataram valores médios de 0,011 e 0,010 folhas $^{-1}$ perfilho $^{-1}$ dia; 5,38 e 5,31 $\mathrm{cm} \mathrm{dia}^{-1}$ perfilho $^{-1}$ e 40,5 e 33,2 cm para o tamanho médio de folhas, respectivamente para períodos de descanso de 36 e 48 dias. O NFV, constante a partir do momento em que a TSF se iguala a TAF, constitui critério objetivo e prático para a definição dos períodos de descanso na lotação rotativa e a intensidade de pastejo na lotação contínua (Santos et al., 2004, Costa et al., 2014).

Para pastagens de M. maximus cv. Mombaça, Costa (2004) recomenda o início do pastejo quando os perfilhos apresentarem, em média, 3 a 4 folhas vivas, de modo a potencializar a produtividade e a qualidade da forragem em oferta. A TEF apresenta alta correlação com a produção de MSV e tem sido utilizada como critério para a seleção de gramíneas em trabalhos de melhoramento genético (Nabinger and Carvalho, 2009). A TEF está diretamente correlacionada com o CMF, pois folhas menores estão normalmente associadas a maiores valores de TAF. Neste trabalho a correlação entre TEF e rendimento de MSV foi positiva e significativa ( $\mathrm{r}$ $=0,891 ; \mathrm{P}<0,05)$; enquanto que com a TAF a 
correlação foi negativa e não significativa $(\mathrm{r}=$ 0,621 ; P > 0,05). A TEF explicou em $81,2 \%$ os incrementos verificados nos rendimentos de MSV, em função do período de descanso. A TAF é a característica morfogênica mais relevante, uma vez que afeta diretamente as três características estruturais do relvado: tamanho da folha, densidade de perfilhos e número de folhas perfilho $^{-1}$ (Pedreira et al., 2009, Santos et al., 2012). Para Difante et al. (2011) a TAF e a TEF apresentam, geralmente, correlação negativa, indicando que quanto maior a TAF, menor será o tempo disponível para o alongamento foliar (Nabinger and Carvalho, 2009).

Tabela 2. Número de folhas vivas (NFV), taxa de aparecimento de folhas (TAF - folhas dia $^{-1}$ perfilho $^{-1}$ ), taxa de expansão foliar ( $\mathrm{TEF}-\mathrm{cm} \mathrm{dia}^{-1}$ perfilho-1 $^{-1}$, comprimento médio de folhas (CMF - cm), índice de área foliar (IAF) e taxa de senescência foliar (TSF - cm dia ${ }^{-1}$ perfilho-1) de Megathyrsus maximus cv. Mombaça, em função do período de descanso.

\begin{tabular}{|c|c|c|c|c|c|c|c|}
\hline \multirow{2}{*}{ Variáveis } & \multicolumn{6}{|c|}{ Períodos de Descanso (dias) } & \multirow{2}{*}{ Equação de Regressão } \\
\hline & 14 & 21 & 28 & 35 & 42 & 49 & \\
\hline NFV & 2,76 & 3,17 & 3,95 & 4,63 & 4,88 & 4,17 & $Y=0,1056+0,2288 X-0,001056 X^{2}\left(R^{2}=0,90\right)$ \\
\hline TAF & 0,197 & 0,151 & 0,141 & 0,132 & 0,116 & 0,085 & $Y=0,2236-0,00272 X\left(r^{2}=0,92\right)$ \\
\hline TEF & 4,08 & 3,92 & 3,81 & 3,08 & 3,61 & 2,78 & $Y=4,5996-0,0334 X\left(r^{2}=0,90\right)$ \\
\hline $\mathrm{CMF}$ & 20,71 & 25,99 & 28,21 & 30,17 & 31,08 & 29,17 & $Y=7,152+1,1887 X-0,0152 X^{2}\left(R^{2}=0,91\right)$ \\
\hline IAF & 1,05 & 1,59 & 2,67 & 3,35 & 3,11 & 3,01 & $Y=2,095+0,2561 X-0,0031 X^{2}\left(R^{2}=0,94\right)$ \\
\hline TSF & 0,087 & 0,107 & 0,135 & 0,154 & 0,166 & 0,198 & $Y=0,0446+0,00319 X\left(r^{2}=0,96\right)$ \\
\hline
\end{tabular}

$\mathrm{O}$ período de descanso afetou positiva e linearmente a TSF (Tabela 2). A senescência foliar decorre da competição por metabólitos e nutrientes entre as folhas velhas e as jovens em crescimento, o que reduz a disponibilidade de forragem de boa qualidade (Santos et al., 2004, Lemaire et al., 2011). Os valores registrados foram inferiores aos reportados por Costa (2004) para $M$. maximus cv. Tanzânia $(0,181 ; 0,199$ e $0,223 \mathrm{~cm}$ dia $^{-1}$ perfilho $^{-1}$, respectivamente para períodos de descanso de 21, 35 e 42 dias). A senescência é o processo natural que caracteriza a última fase de desenvolvimento da folha, iniciado após sua completa expansão e acentuado progressivamente com o aumento da área foliar, em decorrência do sombreamento das folhas inseridas na porção inferior e do baixo suprimento de radiação fotossinteticamente ativa, além de forte competição por luz, nutrientes e água entre os diversos estratos da planta (Nabinger and Pontes, 2002). Quando o perfilho atinge determinado NFV ocorre equilíbrio entre a TAF e a senescência das folhas que ultrapassaram seu período de duração de vida, de modo que o surgimento de nova folha implica na senescência da folha que a precedeu, mantendo o NFV relativamente constante (Lemaire et al., 2011). A senescência afeta negativamente a qualidade da forragem, contudo representa importante processo fisiológico no fluxo de tecidos da gramínea, pois cerca de 35; 68; 86 e $42 \%$ do nitrogênio, fósforo, potássio e magnésio, respectivamente, podem ser reciclados das folhas senescentes e utilizados para a produção de novos tecidos foliares (Sarmiento et al., 2006, Costa et al., 2013).

\section{Conclusões}

O aumento do período de descanso favorece os rendimentos de forragem e o vigor de rebrota, contudo reduz os teores de N, P, Ca, Mg e K. As taxas de aparecimento e expansão de folhas são inversamente proporcionais aos períodos de descanso, ocorrendo o inverso quanto ao comprimento médio de folhas, índice de área foliar e taxa de senescência foliar. O período de descanso mais adequado para pastagens de $M$. maximus cv. Mombaça, visando a conciliar produção, vigor de rebrota e qualidade da forragem, situa-se entre 35 e 42 dias.

\section{Referências Bibliográficas}

Cândido, M. J. D., Alexandrino, E. \& Gomide, J. A. 2005a. Duração do período de descanso e crescimento do dossel de Panicum maximum cv. Mombaça sob lotação intermitente. Revista Brasileira de Zootecnia, 34, 398-405.

Cândido, M. J. D., Gomide, C. A. M., Alexandrino, E., Gomide, J. A. \& Pereira, W. E. 2005b. Morfofisiologia do dossel de Panicum maximum cv. Mombaça sob lotação intermitente com três períodos de descanso. Revista Brasileira de Zootecnia, 34, 406-415. 
Costa, C. A. A., Andrade, A. C., Magalhães, J. A., Mehl, H. U., Rodrigues, B. H. N., da Silva, E. M., Bitencourt, A. B., Santos, F. \& Costa, N. de L. 2014. Características agronômicas dos capins Digitaria sp. e Cynodon dactylon cv. Tifton-85 sob diferentes alturas de resíduo. PubVet, 7, 1509.

Costa, N. L. 2004. Formação, manejo $e$ recuperação de pastagens em Rondônia. Porto Velho, Embrapa Rondonia. 217p.

Costa, N. L., Magalhães, J. A., Pereira, R. G. A., Townsend, C. R. \& Oliveira, J. R. C. 2007. Considerações sobre o manejo de pastagens na Amazônia Ocidental. Revista do Conselho Federal de Medicina Veterinária, 40, 37-56.

Costa, N. L., Moraes, A., Monteiro, A. L. G., Motta, A. C. V., Oliveira, R. A. \& Rodrigues, A. N. A. 2013. Forage productivity and morphogenesis of Axonopus aureus under different nitrogen fertilization rates. Revista Brasileira de Zootecnia, 42, 541-548.

Cunha, F. F., Mota Ramos, M., Brasileiro, A. C. A., Alves , O., Rubens, Cóser, A. C., Martins, C. E., Cecon, P. R. \& Silva, A. R. A. 2012. Produtividade da Brachiaria brizantha cv. Xaraés em diferentes manejos e doses de adubação, períodos de descanso e épocas do ano. Idesia (Arica), 30, 75-82.

Difante, G. S., Nascimento Júnior, D., Silva, S. C., Euclides, V. P. B. \& Montagner, D. B. 2011. Características morfogênicas e estruturais do capim-marandu submetido a combinações de alturas e intervalos de corte. Revista Brasileira de Zootecnia, 40, 955-963.

Gomide, C. A. M., Gomide, J. A. \& Alexandrino, E. 2007. Características estruturais e produção de forragem em pastos de capim-mombaça submetidos a períodos de descanso. Pesquisa Agropecuária Brasileira, 42, 1487-1494.

Lemaire, G., Hodgson, J. \& Chabbi, A. 2011. Grassland productivity and ecosystem services. Cabi, Wallingford. 287p.

Macedo, C. H. O., Alexandrino, E., Jakelaitis, A., Vaz, R. G. M. V., Reis, R. H. P. \& Vendrusculo, J. 2010. Características agronômicas, morfogênicas e estruturais do capim" Panicum maximum" cv. Mombaça sob desfolhação intermitente. Revista Brasileira de Saúde e Produção Animal, 11, 941-952.

Nabinger, C. \& Carvalho, P. C. F. 2009. Ecofisiología de sistemas pastoriles: aplicaciones para su sustentabilidad. Agrociencia, 13, 18-27.

Nabinger, C. \& Pontes, L. S. 2002. Manejo da desfolha. Simpósio sobre manejo da pastagem. FEALQ, Jaboticabal. p.133-158.

Oliveira, P. S. R., Castagnara, D. D., Júnior Gonçalves, A. C., Mesquita, E. E. \& Neres, M. A. 2009. Teores de macrominerais em Panicum maximum cvs. Massai e Mombaça. Synergismus Scyentifica, 4, 7-10

Pedreira, B., Pedreira, C. G. S. \& Silva, S. C. 2009. Acúmulo de forragem durante a rebrotação de capim-xaraés submetido a três estratégias de desfolhação. Revista Brasileira de Zootecnia, 38, 618-625.

Santos, M. R., Fonseca, D. M., Gomes, V. M., Silva, S. P., Silva, G. P. \& Reis, M. 2012. Correlações entre características morfogênicas e estruturais em pastos de capim-braquiária. Ciência Animal Brasileira, 13, 49-56.

Santos, P. M., Balsalobre, M. A. A. \& Corsi, M. 2004. Características morfogenéticas e taxa de acúmulo de forragem do capim-mombaça submetido a três intervalos de pastejo. Revista Brasileira de Zootecnia, 33, 843-851.

Sarmiento, G., Silva, M. P., Naranjo, M. E. \& Pinillos, M. 2006. Nitrogen and phosphorus as limiting factors for growth and primary production in a flooded savanna in the Venezuelan Llanos. Journal of Tropical Ecology, 22, 203-212.

Article History:

Received 21 June 2017

Accepted 20 July 2017

Available on line 8 September 2017

License information: This is an open-access article distributed under the terms of the Creative Commons Attribution License 4.0, which permits unrestricted use, distribution, and reproduction in any medium, provided the original work is properly cited. 